\title{
How to use implantable loop recorders in clinical trials and hybrid therapy
}

\author{
Werner Jung • Vlada Zvereva • Andreas Rillig • \\ Birge Roggenbuck • Gholam Sadeghzadeh • \\ Johannes Kohler
}

Received: 6 February 2011 /Accepted: 28 July 2011 /Published online: 13 October 2011

(C) The Author(s) 2011. This article is published with open access at Springerlink.com

\begin{abstract}
Epidemiological studies show that atrial fibrillation (AF) is associated with a doubling of mortality, even after adjustment for confounders. AF can be asymptomatic, but this does not decrease the thromboembolic risk of the patient. Office ECGs, occasional 24-h Holter recordings and long-term ECG event recording might not be sensitive and accurate enough in patients with AF, especially in those with paroxysmal episodes. In one study, 7 days of continuous monitoring with event recorders detected paroxysmal AF in 20 of 65 patients with a previous negative 24-h Holter recording. Over the last decade, enormous improvements have been made in the technology of implantable devices, which can now store significant information regarding heart rhythm. The first subcutaneous implantable monitor (Reveal XT, Medtronic) was validated for continuous AF monitoring by the XPECT study. The dedicated AF detection algorithm uses irregularity and incoherence of $\mathrm{R}-\mathrm{R}$ intervals to identify and classify patterns in ventricular conduction. Its sensitivity in identifying patients with $\mathrm{AF}$ is $>96 \%$. Numerous clinical data from continuous monitoring of $\mathrm{AF}$ have recently been published. The first applications of this technology have been in the field of surgical and catheter AF ablation. With regard to cryptogenic stroke, an international randomized trial is ongoing to compare standard care with standard care plus the implantable cardiac monitor for $\mathrm{AF}$ detection in patients discharged with the diagnosis of cryptogenic stroke: the Crystal AF trial. Continuous AF monitoring
\end{abstract}

W. Jung $(\bowtie) \cdot$ V. Zvereva $\cdot$ A. Rillig $\cdot$ B. Roggenbuck

G. Sadeghzadeh $\cdot$ J. Kohler

Department of Cardiology,

Academic Teaching Hospital of the University of Freiburg,

Schwarzwald-Baar Klinikum, Vöhrenbacher Street 23,

78050 Villingen-Schwenningen, Germany

e-mail: werner.jung@sbk-vs.de provides an optimal picture of daily $\mathrm{AF}$ burden, both symptomatic and asymptomatic. Implantable cardiac monitors have high sensitivity, enable better assessment of therapy success and may guide further AF therapy.

Keywords Implantable loop recorder Atrial fibrillation . $\mathrm{AF}$ monitoring $\cdot$ Cardiac monitoring

\section{Introduction}

Almost $1-2 \%$ of the general population suffers from atrial fibrillation (AF), and the prevalence is expected to double or triple within the next two to three decades, both in Europe and in the USA [1-4]. Epidemiological observations suggest that $\mathrm{AF}$ is associated with a doubling of mortality, even after adjustment for confounders [2, 5]. Moreover, $\mathrm{AF}$ is the single most important risk factor for ischemic stroke [4]. Therapeutic strategies are aimed at restoring sinus rhythm, controlling the ventricular rate, preventing thromboembolism and minimizing symptoms.

On the other hand, AF can be asymptomatic, but this does not decrease the thromboembolic risk of the patient [6]. Absence of symptoms can even increase this risk if objective information on AF status is lacking, since therapeutic decisions are usually based on both the absence of clinical symptoms and documentation of sinus rhythm.

Office ECGs, occasional 24-h Holter recordings and long-term ECG event recording are the monitoring strategies usually adopted, but they might not be sensitive and accurate enough in patients with AF, especially in those with paroxysmal episodes [7, 8]. Considerable technological advances have been made in the field of 30-day event recorders [9]; however, the use of these devices should be limited to highly compliant patients with frequent symp- 
toms and frequent arrhythmic episodes. These selection criteria, which have also been suggested by recent guidelines on syncope, make external and implantable monitors complementary [10]. Remote monitoring may also play a role in heart failure (HF) patients and may help to detect acute HF decompensation before the onset of symptoms. Appropriate early intervention in these patients may reduce hospitalizations and costs [11]. The diagnostic yield of intermittent monitoring methods is limited and is clearly related to the duration of monitoring. Depending on the specific external monitoring method, sensitivity lies between $31.3 \%$ and $71.0 \%$, whereas the negative predictive value (NPV) ranges between $21.5 \%$ and $64.6 \%[7,8]$ (Table 1).

Continuous monitoring of $\mathrm{AF}$ is possible through the diagnostics of implantable dual-chamber pacemakers, dualchamber ICDs or implantable loop recorders (ILR). Given the limitations inherent in intermittent monitoring, accurate quantitative assessment of the Paroxysmal (P) AF burden can only be achieved by using continuous cardiac rhythm monitoring.

\section{Assessment of symptomatic and asymptomatic AF}

While AF can be associated with typical symptoms such as palpitations, dyspnea, dizziness and syncope, a considerable proportion of patients remain asymptomatic. In addition, symptomatic patients can also have asymptomatic episodes; on the other hand, symptoms suggestive of $\mathrm{AF}$ may sometimes not correspond to AF on the ECG. This means that relying on symptoms to drive therapies for $\mathrm{AF}$ can be very misleading. The advent of diagnostic features for automatic AF detection, regardless of symptoms, has further evidenced the burden of asymptomatic AF in the general population suffering from this arrhythmia.

This issue has profound implications for the precise definition of therapeutic success or endpoint of pharmacological prevention, cardioversion and ablation in the management of AF. Thus, the low reliability of patients' symptoms and the limitations of intermittent AF monitoring strategies have dramatically increased the difficulty of decisions regarding patients assumed to be AF-free. The same issue affects the comparison of different therapeutic approaches in clinical trials when symptoms and/or intermittent methods of AF monitoring are used. Intuitively, the results of such comparisons are dependent upon the method of assessment and the population studied; that longer periods of continuous monitoring would increase the detection of asymptomatic AF is supported by clinical studies.

Studies using only electrocardiogram (ECG) recordings during clinic visits have found a $5 \%-20 \%$ prevalence of asymptomatic $\mathrm{AF}$ in unselected patient groups [12, 13], but Holter monitoring and event recorders have been seen to increase the diagnostic yield for asymptomatic AF [14]. In one study, 7 days of continuous monitoring with event recorders detected paroxysmal $\mathrm{AF}$ in 20 of 65 patients with a previous negative 24-h Holter recording [15]. Similarly, Jabaudon et al. [16] showed that the longer we monitor patients who have had a stroke, the more $\mathrm{AF}$ we find. In the Prevention of Atrial Fibrillation After Cardioversion (PAFAC) trial [17], about 70\% of AF episodes detected by transtelephonic transmission were asymptomatic. In patients with previously documented paroxysmal AF, sustained asymptomatic AF may be even more frequent than symptomatic AF. Thus, the high incidence of stroke in the rhythm-control arm of trials such as the AFFIRM study may be related to the withdrawal of anticoagulation therapy for patients believed to be in sinus rhythm [6]. Moreover, there is a lack of correlation among $\mathrm{AF}$ symptoms, heart rate variation at the onset of $\mathrm{AF}$, episode duration, concomitant heart disease and antiarrhythmic drug treatment $[18,19]$.

It is probable that the main determinant of symptoms is the subjective perception of each patient, and this may differ markedly among patients, making assessment unreliable. Patients may perceive AF-like symptoms in the

Table 1 AF monitoring options

\begin{tabular}{|c|c|c|c|c|}
\hline Technology & Storage & Continuous & Electrodes & Comments \\
\hline Symptoms & None & Yes & None & Only symptomatic events \\
\hline ECG & $<1 \min$ & Yes & 10 on skin & \\
\hline Holter & $24-48 \mathrm{~h}$ & Yes & 3 on skin & \\
\hline Event recorder & $7-28$ days & No & 3 on skin & Only symptomatic events \\
\hline Transtelephonic ECG monitoring & $\mathrm{min} /$ day & No & On skin & Discontinuous \\
\hline External loop recorder & $7-28$ days & Yes & On wrist or $2-3$ on skin & \\
\hline Mobile cardiac outpatient monitoring & Continuous, $(<28$ days $)$ & Yes & 3 on skin & Direct transmission \\
\hline Implantable cardiac monitor & Continuous & Yes & Under skin & Implanted \\
\hline Pacemaker, ICD & Continuous & Yes & Implanted & Implanted, PM/ICD pt. \\
\hline
\end{tabular}


absence of arrhythmia. As a consequence, "time to first symptomatic AF recurrence" is not an appropriate endpoint, as it assumes that $\mathrm{AF}$ recurrences are random and $\mathrm{AF}$ is symptomatic.

\section{Ischemic stroke and continuous AF monitoring}

Over the last decade, enormous improvements have been made in the technology of implantable devices, which can now store significant information regarding heart rhythm, and numerous clinical data on the continuous monitoring of AF have recently been published. This technology now enables in vivo patterns of human $\mathrm{AF}$ to be reconstructed, allowing a novel insight into the natural history and pathophysiology of this arrhythmia over a long period [20]. It can show both the progression of the disease from the paroxysmal to the persistent form and the success of treatment.

The correlation between the presence of atrial tachyarrhythmias during chronic monitoring and clinical outcomes was first evaluated in the MOST trial [21] in a subgroup of 312 patients receiving pacemakers for sinus node dysfunction. The presence of atrial high rate episodes $(\geq 220$ beats/ min and longer than $5 \mathrm{~min}$ ) was found to be an independent predictor of total mortality, as well as death or nonfatal stroke. This does not mean that $5 \mathrm{~min}$ of AF is dangerous; the authors did not perform any analysis to correlate the duration of the episodes with the clinical events. This threshold was the result of a technical compromise in order to exclude artifacts and store only atrial arrhythmias.

Capucci et al. [22] reported that patients with devicedetected AF recurrences longer than 1 day had a 3.1-fold higher risk of embolism than patients without, or with shorter, AF recurrences, a finding that indicates that $\mathrm{AF}$ recurrences longer than 1 day are independently associated with arterial embolism. Similar findings were reported by Botto et al. [8], who also showed that the duration of AF per day should be considered in combination with the risk factors for stroke, as summarized by the $\mathrm{CHADS}_{2}$ score. $\mathrm{AF}$ episodes lasting more than $24 \mathrm{~h}$ can be dangerous in patients with a score $=1$, while this is not the case for shorter episodes. However, Botto et al. did not analyze the data in such a way as to establish a threshold of the daily AF burden (hours in 1 day) above which danger is incurred; rather, they only analyzed events in patients with AF lasting more or less than $24 \mathrm{~h}$.

The TRENDS study [23] tried to assess this threshold. The TRENDS data suggest that $\mathrm{AF}$ burden $\geq 5.5 \mathrm{~h}$ on any day in the last 30 days is associated with an approximate doubling of the risk of thromboembolism compared with zero AF burden, after controlling for clinical stroke risk factors and antithrombotic use.
The multicenter, large-scale, ongoing IMPACT trial [24] was designed to test the hypothesis that initiation and withdrawal of oral anticoagulant therapy guided by continuous monitoring improve clinical outcomes by reducing the combined rate of stroke, systemic embolism and major bleeding more effectively than conventional clinical management. The early detection of AF generates an automatic alert to initiate anticoagulation based on patient-specific stroke risk stratification. Subsequently, freedom from AF for predefined periods prompts withdrawal of anticoagulation in order to avoid bleeding.

\section{Subcutaneous leadless AF monitoring}

Recently, the first subcutaneous ILR was validated for continuous AF monitoring by the XPECT study [25]. The ICM (Reveal XT; Medtronic Inc.) is equipped with a new AF detection algorithm that is designed to detect the presence of $\mathrm{AF}$ episodes and to quantify the AF burden. In addition, the ICM features detection algorithms for bradyarrhythmias and ventricular tachyarrhythmias. Physiological sinus rhythm and AF each have a unique R-R interval pattern. The dedicated AF detection algorithm uses irregularity and incoherence of $\mathrm{R}-\mathrm{R}$ intervals to identify and classify patterns in ventricular conduction. The R-R intervals are analyzed within each 2min period, and the difference in duration between consecutive $R-R$ intervals $(\Delta R-R)$ is calculated.

Subsequently, the variability of these $\Delta \mathrm{R}-\mathrm{R}$ intervals is calculated, much as a Lorenz plot is constructed. When the $\mathrm{R}-\mathrm{R}$ intervals within the 2-min period show a certain pattern of irregularity, the heart rhythm in this period is classified as AF. If $\mathrm{R}-\mathrm{R}$ intervals are regular, with some sinus node modulations, the 2 -min period is defined as normal sinus rhythm (Fig. 1). The ICM can store up to $49.5 \mathrm{~min}$ of recorded ECG, which is allocated to $27 \mathrm{~min}$ of automatically activated events and $22.5 \mathrm{~min}$ of patientactivated events. In addition, the ICM has an episode log that can catalogue 30 automatically detected AF episodes and up to ten patient-activated episodes. When the memory is full, an additional episode will overwrite the oldest stored episode. The devices can be implanted subcutaneously under local anesthesia.

The XPECT study compared the diagnostic data stored automatically by the ICM with the corresponding visual inspection of the ECG traces carried out by the investigators. The study showed that the overall accuracy of the ICM in measuring AF burden was $98.5 \%$, with a Pearson correlation coefficient of $r=0.976$ between the daily burden automatically calculated by the device and that measured by the investigators. The sensitivity in identifying patients with any AF was $96.1 \%$ and specificity $85.4 \%$. The corresponding PPV and NPV were $79.3 \%$ and $97.4 \%$, respectively. 
Fig. 1 R-R intervals are analyzed within each 2-min period, and the difference in duration between consecutive $\mathrm{R}-\mathrm{R}$ intervals $(\Delta R-R)$ is calculated. The variability of $\Delta R-R$ intervals is calculated in order to distinguish sinus rhythm (left side) from atrial fibrillation (right side)

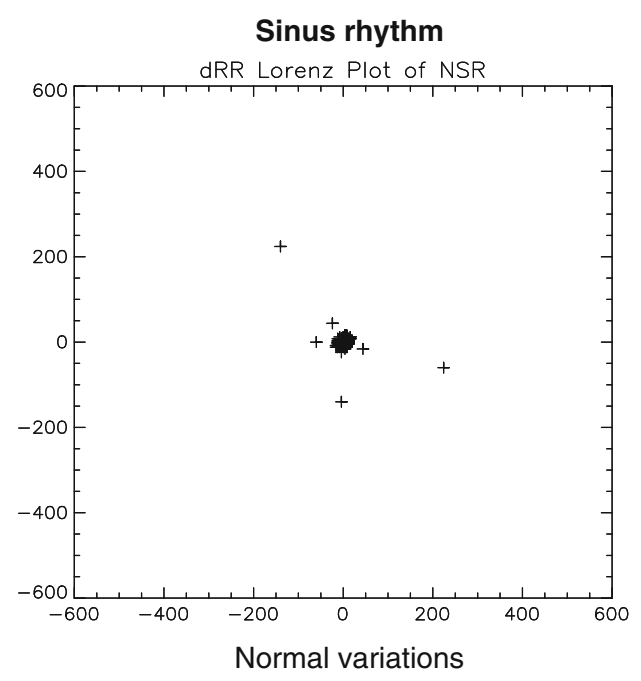

\section{Recent trials with subcutaneous AF monitoring and future perspectives}

The first experience of using a permanently implantable ICM, the Reveal XT (Medtronic, Inc.), following surgical therapy for AF was reported by Hanke et al. [26]. The aims of the study were to accurately evaluate the evolution of heart rhythm after surgical ablation therapy and to examine potential discrepancies in identifying post-procedural AF recurrence by means of intra-patient comparison of two different follow-up strategies: conventional 24-h Holter monitoring (24HM) at prescheduled times and continuous cardiac rhythm surveillance. Continuous heart rhythm surveillance was much more accurate in detecting AF recurrence after surgical ablation therapy. In addition, the study provided strong evidence that the commonly used intermittent follow-up strategy is significantly inferior to full-disclosure heart rhythm observation with respect to AF recurrence detection. Hanke et al. demonstrated that, even when 24HM was performed quarterly, the level of correct interpretation, in comparison with that of continuous heart rhythm surveillance, was still unsatisfactory, sensitivity being only $60 \%$.

Pokushalov et al. [27] used the ILR for continuous AF monitoring in 89 patients with symptomatic, drug-refractory, longstanding persistent AF, who had undergone anatomic ablation of the areas of ganglionated plexi in the left atrium. In 29 patients, a second procedure for circumferential pulmonary vein isolation was performed, and five of them were subjected to a third circumferential ablation procedure. After a followup of $16 \pm 7$ months from last procedure, 53 (59.6\%) of the 89 patients were in sinus rhythm, as documented by the ILR, without antiarrhythmic drug therapy. The authors found that the long-term success rate in patients who underwent a single ablation procedure involving ganglionated plexi ablation alone was $38.2 \%$ over a follow-up of $24 \pm 3$ months. Using the same monitoring technique, the same group [28] showed that ablation of the areas of the ganglionated plexi in 56 patients with paroxysmal AF yielded a success rate of $71 \%$ over a 12-month period.
Fig. 2 This is an example from the Cardiac Compass, the software tool provided by the programmer to show the trend in data during follow-up. The trend in the daily AF burden shows that paroxysmal AF is worsening over time, with longer and longer episodes. The ECG strip stored by Reveal XT shows the onset of an AF episode

\section{Trend of daily AF burden from Cardiac Compass}

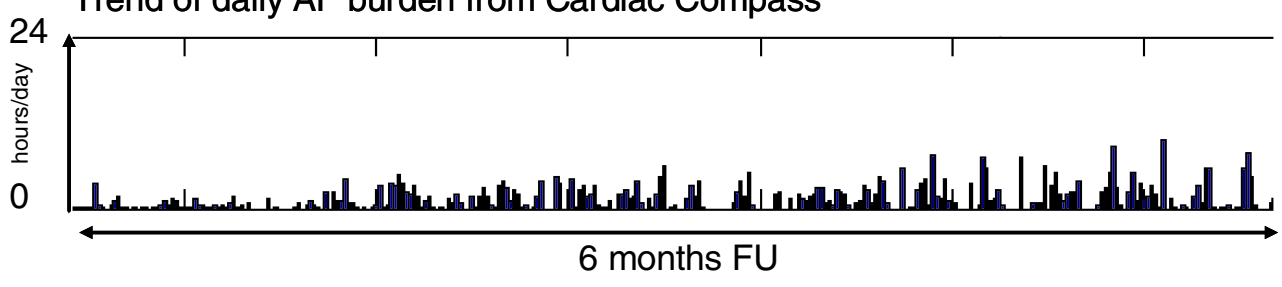

ECG at the onset of $A F$, as stored by the ICM

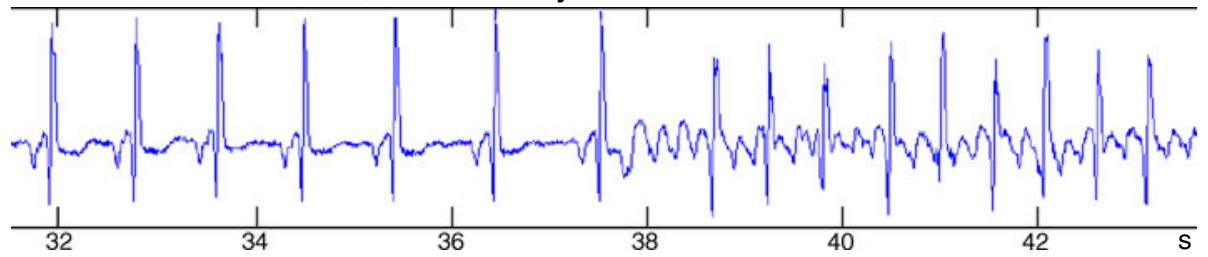


Reveal XT has also been used to compare preablation and post-ablation $\mathrm{AF}$ burden in patients with paroxysmal or persistent AF [29]; ablation dramatically and significantly reduced $\mathrm{AF}$ burden, and $62 \%$ of patients were AF-free 9 months after the procedure. Similar results were also obtained by Amellone et al. [30] and Chun et al. [31] through continuous subcutaneous postablation monitoring.

In the field of cryptogenic stroke, an international randomized trial is ongoing to compare standard care (SC) with SC plus the Reveal XT ICM for AF detection in patients discharged with the diagnosis of cryptogenic stroke: the Crystal AF trial [32].

For pharmaceutical companies, the use of continuous $\mathrm{AF}$ monitoring in $\mathrm{AF}$ trials is particularly advantageous; as all AF episodes will be registered, fewer patients will be required and the effect of therapy will become apparent in a shorter period of time. One of the first pilot studies was performed by Arya et al., [33], who evaluated a new AF drug (budiodarone) in pacemaker patients. A direct correlation between $\mathrm{AF}$ burden and drug dosage was observed and $\mathrm{AF}$ burden returned to baseline values during drug washout.

In the future, any AF trial studying the effect of a rhythm control intervention should consider continuous monitoring as the elective tool for reliably measuring the effect of therapy. Figure 2 shows an example of the trend in the daily AF burden recorded by a Reveal XT (Medtronic) device implanted in a patient with paroxysmal AF. The data clearly show progression of the disease and the ECG allows validation by the physician. This rigorous approach is also confirmed in the Consensus Statement of the EHRA and the German AFNET [34], according to which, with regard to ECG-based outcomes, "To assess freedom from AF, continuous ECG recording is the gold standard."

\section{Conclusions}

Symptom-related or intermittent AF measurement in $\mathrm{AF}$ trials is highly unreliable in the evaluation of a rhythm control intervention. Continuous AF monitoring provides an optimal picture of both symptomatic and asymptomatic daily AF burden.

Implantable cardiac monitors have high sensitivity, provide a better assessment of therapy success, and may guide further AF therapy.

Acknowledgment The author thanks Alphons Vincent of Medtronic Europe and Giorgio Corbucci of Medtronic Bakken Research Center for their support.

\section{Conflict of interest None.}

Open Access This article is distributed under the terms of the Creative Commons Attribution Noncommercial License which permits any noncommercial use, distribution, and reproduction in any medium, provided the original author(s) and source are credited.

\section{References}

1. Stewart, S., Hart, C. L., Hole, D. J., \& McMurray, J. J. (2001). Population prevalence, incidence, and predictors of atrial fibrillation in the Renfrew/Paisley study. Heart, 86, 516-521.

2. Stewart, S., Hart, C. L., Hole, D. J., \& McMurray, J. J. (2002). A population-based study of the long-term risks associated with atrial fibrillation: 20-year follow-up of the Renfrew/Paisley study. American Journal of Medicine, 113, 359-364.

3. Miyasaka, Y., Barnes, M. E., Gersh, B. J., Cha, S. S., Bailey, K. R., Abhayaratna, W. P., et al. (2000). Secular trends in incidence of atrial fibrillation in Olmsted County, Minnesota, 1980 to 2000, and implications on the projections for future prevalence. Circulation, 114, 119-125.

4. Camm, A. J., Kirchhof, P., Lip, G. Y., Schotten, U., Savelieva, I., Ernst, S., et al. (2010). Guidelines for the management of atrial fibrillation: the Task Force for the Management of Atrial Fibrillation of the European Society of Cardiology (ESC). Developed with the special contribution of the European Heart Rhythm Association (EHRA); Endorsed by the European Association for Cardio-Thoracic Surgery (EACTS). European Heart Journal, Aug 29 [Epub ahead of print].

5. Benjamin, E. J., Wolf, P. A., D’Agostino, R. B., Silbershatz, H., Kannel, W. B., \& Levy, D. (1998). Impact of atrial fibrillation on the risk of death: the Framingham Heart Study. Circulation, 98, 946-952.

6. Flaker, G. C., Belew, K., Beckman, K., Vidaillet, H., Kron, J., Safford, R., et al. (2005). Asymptomatic atrial fibrillation: Demographic features and prognostic information from the Atrial Fibrillation Follow-up Investigation of Rhythm Management (AFFIRM) study. American Heart Journal, 149, 657-663.

7. Ziegler, P. D., Koehler, J. L., \& Mehra, R. (2006). Comparison of continuous versus intermittent monitoring of atrial arrhythmias. Heart Rhythm, 3, 1445-1452.

8. Botto, G. L., Padeletti, L., Santini, M., Capucci, A., Gulizia, M., Zolezzi, F., et al. (2009). Presence and duration of atrial fibrillation detected by continuous monitoring: crucial implications for the risk of thromboembolic events. Journal of Cardiovascular Electrophysiology, 20, 241-248.

9. Olson, J. A., Fouts, A. M., Padanilam, B. J., \& Prystowsky, E. N. (2007). Utility of mobile cardiac outpatient telemetry for the diagnosis of palpitations, presyncope, syncope, and the assessment of therapy efficacy. Journal of Cardiovascular Electrophysiology, 18, 473-477.

10. Task Force for the Diagnosis and Management of Syncope; European Society of Cardiology (ESC); European Heart Rhythm Association (EHRA); Heart Failure Association (HFA); Heart Rhythm Society (HRS), Moya, A., Sutton, R., Ammirati, F., Blanc, J. J., Brignole, M., et al. (2009). Guidelines for the diagnosis and management of syncope (version 2009). European Heart Journal, 30(21), 2631-1671.

11. Anand, I. S., Greenberg, B. H., Fogoros, R. N., Libbus, I., Katra, R. P., \& Investigators, M. (2011). Design of the Multi-Sensor Monitoring in Congestive Heart Failure (MUSIC) study: prospective trial to assess the utility of continuous wireless physiologic monitoring in heart failure. Journal of Cardiac Failure, 17(1), 1116. 
12. Psaty, B. M., Manolio, T. A., Kuller, L. H., et al. (1997). Incidence of and risk factors for atrial fibrillation in older adults. Circulation, 96, 2455-2461.

13. Furberg, C. D., Psaty, B. M., Manolio, T. A., Gardin, J. M., Smith, V. E., \& Rautaharju, P. M. (1994). Prevalence of atrial fibrillation in elderly subjects (the Cardiovascular Health Study). American Journal of Cardiology, 74, 236-241.

14. Kinlay, S., Leitch, J. W., Neil, A., Chapman, B. L., Hardy, D. B., \& Fletcher, P. J. (1996). Cardiac event recorders yield more diagnoses and are more cost-effective than 48-hour Holter monitoring in patients with palpitations. Annals of Internal Medicine, 124, 16-20.

15. Roche, F., Gaspoz, J. M., Da Costa, A., et al. (2005). Frequent and prolonged asymptomatic episodes of paroxysmal atrial fibrillation revealed by automatic long-term event recorders in patients with a negative 24-hour Holter. Pacing and Clinical Electrophysiology, $25,1587-1593$.

16. Jabaudon, D., Sztajzel, J., Sievert, K., Landis, T., \& Sztajzel, R. (2004). Usefulness of ambulatory 7-day ECG monitoring for the detection of atrial fibrillation and flutter after acute stroke and transient ischemic attack. Stroke, 35, 1647-1651.

17. Fetsch, T., Bauer, P., Engberding, R., et al. (2004). Prevention of atrial fibrillation after cardioversion: results of the PAFAC trial. European Heart Journal, 25, 1385-1394.

18. Israel, C. W., Gronefeld, G., Ehrlich, J. R., Li, Y. G., \& Hohnloser, S. H. (2004). Long-term risk of recurrent atrial fibrillation as documented by an implantable monitoring device: implications for optimal patient care. Journal of the Am College of Cardiologists, 43, 47-52.

19. Quirino, G., Giammaria, M., Corbucci, G., Pistelli, P., Turri, E., Mazza, A., et al. (2009). Diagnosis of paroxysmal atrial fibrillation in patients with implanted pacemakers: relationship to symptoms and other variables. Pacing and Clinical Electrophysiology, 32, 91-98.

20. Saksena, S., Hettrick, D. A., Koehler, J. L., Grammatico, A., \& Padeletti, L. (2007). Progression of paroxysmal atrial fibrillation to persistent atrial fibrillation in patients with bradyarrhythmias. American Heart Journal, 154(5), 884-892.

21. Glotzer, T. V., Hellkamp, A. S., Zimmerman, J., Sweeney, M. O., Yee, R., Marinchak, R., et al. (2003). Atrial high rate episodes detected by pacemaker diagnostics predict death and stroke. Circulation, 107, 1614-1619.

22. Capucci, A., Santini, M., Padeletti, L., Gulizia, M., Botto, G., Boriani, G., et al. (2005). Monitored atrial fibrillation duration predicts arterial embolic events in patients suffering from bradycardia and atrial fibrillation implanted with antitachycardia pacemakers. Journal of the Am College of Cardiologists, 46, 1913-1920.

23. Glotzer, T. V., Daoud, E. G., Wyse, D. G., Singer, D. E., Ezekowitz, M. D., Hilker, C., et al. (2009). The relationship between daily atrial tachyarrhythmia burden from implantable device diagnostics and stroke risk. The TRENDS Study. Circulation: Arrhythmias and Electrophysiology, 2, 474-480.
24. Ip, J., Waldo, A. L., Lip, G. Y. H., Rothwell, P. M., Martin, D. T., Bersohn, M. M., et al. (2009). Multicenter randomized study of anticoagulation guided by remote rhythm monitoring in patients with implantable cardioverter-defibrillator and CRT-D devices: Rationale, design, and clinical characteristics of the initially enrolled cohort: the IMPACT study. American Heart Journal, 158, 364-370.e1.

25. Hindricks, G., Pokushalov, E., Urban, L., Taborsky, M., Kuck, K.H., Lebedev, D., et al. on behalf of the XPECT Trial investigators (2010). Performance of a new leadless implantable cardiac monitor in detecting and quantifying atrial fibrillation. Results of the XPECT Trial. Circulation: Arrhythmias and Electrophysiology, 3, 141-147.

26. Hanke, T., Charitos, E. I., Stierle, U., Karluss, A., Kraatz, E., Graf, B., et al. (2009). Twenty-four-hour holter monitor follow-up does not provide accurate heart rhythm status after surgical atrial fibrillation ablation therapy: up to 12 months experience with a novel permanently implantable heart rhythm monitor device. Circulation, 120(11 Suppl), S177-S184.

27. Pokushalov, E., Romanov, A., Artyomenko, S., Turov, A., Shugayev, P., Shirokova, N., et al. (2010). Ganglionated plexi ablation for longstanding persistent atrial fibrillation. Europace, 12, 342-346.

28. Pokushalov, E., Romanov, A., Artyomenko, S., Turov, A., Shirokova, N., \& Katritsis, D. G. (2010). Left atrial ablation at the anatomic areas of ganglionated plexi for paroxysmal atrial fibrillation. Pacing and Clinical Electrophysiology, 33(10), 12311238 .

29. Corbucci, G., Urban, L., Hatala, R., Jongen, T., De Melis, M. (2010). Reliability of atrial fibrillation burden through subcutaneous devices. Europace, 12(suppl 1), abstract 1811.

30. Amellone, C., Giuggia, M., Trapani, G., Giordano, B., Ceresa, M., Fazzari, M., et al. (2010). Rhythm surveillance after atrial fibrillation ablation: comparison between conventional follow up and a novel implantable continuous cardiac rhythm monitoring device. Heart Rhythm, 7(5) May Supplement, AB04-AB05.

31. Chun, K. R., Köster, I., Metzner, A., Fürnkranz, A., Schmidt, B., Ouyang, F., et al. (2010). Cryoballoon applications after acute pulmonary vein isolation - the Alster Extra Study. Heart Rhythm, 7(5) May Supplement: P06-P49.

32. Sinha, A. M., Diener, H. C., Morillo, C. A., Sanna, T., Bernstein, R. A., Di Lazzaro, V., et al. (2010). Cryptogenic Stroke and underlying Atrial Fibrillation (CRYSTAL AF): design and rationale. American Heart Journal, 160, 36-41.

33. Arya, A., Silberbauer, J., Teichman, S. L., Milner, P., Sulke, N., \& Camm, A. J. (2009). A preliminary assessment of the effects of ATI-2042 in subjects with paroxysmal atrial fibrillation using implanted pacemaker methodology. Europace, 11(4), 458-464.

34. Kirchhof, P., Bax, J., Blomstrom-Lundquist, C., Calkins, H., Camm, A. J., Cappato, R., et al. (2009). Early and comprehensive management of atrial fibrillation: proceedings from the II AFNET/ EHRA consensus conference on atrial fibrillation entitled 'research perspectives in atrial fibrillation. Europace, 11, 860-885. 